Check for updates

Cite this: RSC Adv., 2019, 9, 37321

Received 11th October 2019

Accepted 11th November 2019

DOI: 10.1039/c9ra08296d

rsc.li/rsc-advances

\section{Synthesis of a semi-conductor-like MOF with black phosphorous as a composite for visible light-driven photocatalysis $\dagger$}

\author{
Philani Vusumuzi Hlophe and Langelihle Nsikayezwe Dlamini (iD *
}

\section{Introduction}

Environmental remediation and the splitting of water into hydrogen and oxygen using photocatalysis technology has been explored as a cost effective strategy to address environmental problems such as water pollution and production of ecofriendly fuels. ${ }^{1}$ The premise of photocatalysis involves a semiconductor material which when excited by solar irradiation produces electron-hole pairs which are separated and transferred to the conduction band and valence band, where they initiate redox reactions. ${ }^{2}$ Several traditional semiconductors have been utilised such as $\mathrm{TiO}_{2}$ (ref. 3 ) and $\mathrm{ZnO},{ }^{4}$ however these materials are disadvantaged by fast recombination of photogenerated charge transporters which affects their charge separation ability. ${ }^{5}$ In addition to the fast recombination setback, these materials are UV active which invariantly limits their effectual utilization of the solar spectrum and thus they exhibit poor photocatalytic activity. ${ }^{6}$

Due to these drawbacks, current research focus is aimed at fabricating visible light responsive materials with good separation of photo-irradiated electrons and holes. This can be achieved by synthesizing semiconductor composite materials which involves combining the merits of two or more

Department of Chemical Sciences, University of Johannesburg, Doornfontein Campus, P. O. Box 17011, Doornfontein, Johannesburg, 2028, South Africa. E-mail: Indlamini@uj.ac.za

$\dagger$ Electronic supplementary information (ESI) available. See DOI: 10.1039/c9ra08296d semiconductors. These composite materials aim to enhance the charge carrier separation and thus improve the photocatalytic activity. Semiconductor composites rely on staggered alignment of the band edge positions, which ensures that electrons and holes are directed to different units via a junction formed between the semiconductors. ${ }^{7}$ The separation of the charge carriers is vital for redox reactions to occur. ${ }^{2}$ Heterojunctions such as metal oxides@MOFs have been utilized as visible light irradiated materials where the separation of charges was the paramount focus. However in the work reported here, a 2D@MOF composite is fabricated which relies on the effectiveness of black phosphorus as an excellent charge transporter in addition to the separation of charge carriers between the two semiconductor materials..$^{\mathbf{8} 9}$

Metal-Organic Frameworks (MOFs) are a category of translucent substances that comprise of coordinate bonds between metal nodes and organic ligands. ${ }^{\mathbf{1 0}}$ MOFs have come to the fore as a comprehensive set of crystalline materials with well-defined porosity. MOFs are different from other permeable materials, they boast many merits such as well-defined porosity, large surface area, ease of synthesis, thermal stability, ultra-low densities and with extensive properties convenient for physical and chemical implementation. ${ }^{\mathbf{1 1}}$ These intriguing features have triumphed the implementation of MOFs in gas storage, catalysis, segregation of fluids including water purification. ${ }^{12}$

A great amount of research has been done to enhance the necessary conditions for introduction of new or improved functionalities of structural profile and permanent permeable 
framework of MOFs to broaden applications towards magnetic and electronic devices, proton conduction, biomedicine supercapacitors, sensors and rechargeable lithium ion batteries. ${ }^{13}$ Numerous MOF structures with exceptional porosity have been presented and most of them are built from divalent cations such as $\mathrm{Zn}^{2+}, \mathrm{Cd}^{2+}, \mathrm{Cu}^{2+}$. They are constructed from carboxylates, phosphonates or $\mathrm{N}$ contributing spacers, or a composite of all these species. This results in MOFs with different structural conformations and pore sizes, ranging from micro to mesoporous architectures and the organic linker showing or lacking functional groups. MOFs constructed from tri or tetravalent metal ions are uncommon, apart from $\mathrm{Ln}^{3+}$ cations and the newly discovered uranyl moieties..$^{\mathbf{1 4 1 5}}$ This anomaly is a result of chemical reactivity differences compared to lower valence metals.

The titanium-based MOF, MIL-125 (Ti) enjoys a high surface area, which is propitious for organic molecule absorption. A speedy photochromic behaviour is noticed upon UV-visible illumination, when alcohols accumulate on the facet of the MIL-125 (Ti) in a nitrogen atmosphere. This photochromic effect is due to the existence of the intervalence charge transfer, which results from the optically initiated movement of electrons from titanium (III) to titanium (IV) centres in the titanium oxoclusters. However, reoxidation of produced $\mathrm{Ti}^{3+}$ species into $\mathrm{Ti}^{4+}$ species takes place rapidly in the presence of air. This reverse photo-induced oxidation of $\mathrm{Ti}^{3+}$ to $\mathrm{Ti}^{4+}$ can be related to the depletion of $\mathrm{O}_{2}$ into dual-atomic superoxide $\left(\mathrm{O}_{2}{ }^{-}\right)$species, with no structural deformation. ${ }^{\mathbf{1 4}}$

Nevertheless, the photocatalytic efficiency of MIL-125(Ti) has been probed to a restricted degree and it is of great interest to evolve novel visible light driven photocatalysts.

Two dimensional materials have for the last couple of years showed a great potential as visible light responsive photocatalysts. These materials boast remarkable electronic, optical and catalytic properties which has led to their applications in various fields including photocatalytic water splitting. ${ }^{16}$ Examples of these 2D materials include graphene, $\mathrm{MoS}_{2}$ and transition metal dichalcogenides (TMDs). The limitation of graphene is its lack of a band gap, while $\mathrm{MoS}_{2}$ has a low carrier mobility and TMDs have a wide band gap which limits their photocatalytic efficiency of these $2 \mathrm{D}$ photocatalysts. ${ }^{17}$

Black phosphorus (BP), an emerging direct band gap 2D material has come to the fore front in recent years as it bridges the gap between band gapless graphene and wide band gap TMDs. ${ }^{18}$ Black phosphorus is an allotrope of phosphorus alongside white and red phosphorus, however it is more thermodynamically stable than its two counterparts. ${ }^{19}$ This remarkable material boasts intriguing properties such as high charge carrier mobility, low toxicity, an adjustable band gap, spanning from $0.3 \mathrm{eV}$ for bulk and $2.0 \mathrm{eV}$ for monolayer. The tunability of the band gap allows BP to be optimized for the certain applications by exfoliating the bulk intermediate into few layers. ${ }^{20}$ Additionally this p-type semiconducting 2D material also absorbs in the visible and near infrared region, which is propitious for photocatalytic applications. However, the downside of BP is by far it's unstable nature in ambient conditions.
Thus, fabricating a n-MOF/BP composite would greatly improve the stability of the obtained hybrid, in addition to improved photocatalytic performance. In this work we report for the first time the synthesis of a novel visible light MIL125(Ti)/BP photocatalyst, which will exhibit an inhibited recombination of charge carriers.

\section{Materials and methods}

\section{Chemicals}

All regents and solvents utilized were of analytical reagent grade and used as received from suppliers. Terephthalic acid (BDC), $N^{\prime}, N$-dimethylformanide (DMF), $N$-methylpyridine (NMP), sodium hydroxide $(\mathrm{NaOH})$ titanium isopropoxide (TBOT), bulk black phosphorus, ethanol ( $\geq 99.8 \%$ absolute), sodium sulphate ( $\geq 99 \%$ Reagent Plus), polyvinylidene fluoride (PVDF) and methanol were purchased from Sigma-Aldrich Co. Ltd.

\section{Synthesis of MIL-125}

The fabrication of MIL-125(Ti) was done following the synthesis route reported by Yang et $a l .{ }^{21}$ with slight modifications. Typically $2.2 \mathrm{~g}$ of 1,4-benzenedicarboxylic acid was dissolved in a solution of $36 \mathrm{~mL}$ DMF and $4 \mathrm{~mL}$ methanol. A volume of $2.4 \mathrm{~mL}$ titanium isopropoxide was then added to the resultant solution and a white solution was formed, which was then sonicated for $5 \mathrm{~min}$ to ensure a homogeneous dispersion. The sonicated dispersion was exposed to hydrothermal conditions in a $100 \mathrm{~mL}$ Teflon lined autoclave for $24 \mathrm{~h}$ at $150{ }^{\circ} \mathrm{C}$. Post cooling to room temperature, a gel-like solid was secured which was washed once with DMF and twice with methanol. The solid was then dried at room temperature for $24 \mathrm{~h}$ at $60{ }^{\circ} \mathrm{C}$.

\section{Synthesis of FL-BP}

The FL-BP nanosheets were synthesized according to a procedure reported by Zhu et al. ${ }^{22}$ In detail $20 \mathrm{mg}$ of bulk BP was added to a solution of saturated $\mathrm{NaOH} / \mathrm{NMP}$ (the addition of the bulk phosphorus to the $\mathrm{NaOH} / \mathrm{NMP}$ solution was carried out in an argon glovebox to combat the degradation of the bulk material). The saturated $\mathrm{NaOH} / \mathrm{NMP}$ solution was made by dissolving $0.05 \mathrm{~g} \mathrm{NaOH}$ in $20 \mathrm{~mL}$ NMP. This aqueous solution was then sonicated for 4 hours in an ice bath. Post sonication the solution was centrifuged at $2000 \mathrm{rpm}$ for 20 minutes to remove any non-exfoliated bulk material.

\section{Synthesis of MIL-125(Ti)/BP composites}

The composites were synthesized varying the weight loadings of MIL-125(Ti) while keeping the amount of FL-BP constant. Typically, MIL-125(Ti) $\left(1.8 \times 10^{-4}, 3.6 \times 10^{-4}\right.$, and $5.4 \times 10^{-4}$ mol) was added to $10 \mathrm{~mL}$ of FL-BP. This dispersion was then sonicated for 2 hours, after which it was centrifuged and washed with ethanol three times. The wet powders were then dried in an oven at $60{ }^{\circ} \mathrm{C}$ overnight. The composites were denoted 4\%BpMIL, 6\%BpMIL and 12\%BpMIL $(\mathrm{m} / \mathrm{m})$ respectively. 


\section{Characterization techniques}

Morphologies of the nanocomposites were captured using scanning electron microscopy (SEM) and transmission electron microscopy (TEM). The SEM (TESCAN Vega TC) was operated at $20 \mathrm{kV}$ operating voltage under nitrogen gas using VEGA 3 TESCAN software. Elemental composition of the nanoparticles (NPs) was elucidated with the SEM coupled with energy dispersive spectroscopy (EDS) and was operated at $20 \mathrm{kV}$. A TEM which was operated at an accelerating voltage of $200 \mathrm{kV}$ was used to further acquire images of the nanocomposites. The nanocomposites were deposited on carbon coated copper grids after being dispersed in ethanol. Furthermore, selected area electron diffraction (SAED) was conducted on the nanocomposites at 2025k magnification to elucidate their crystal phases.

Powdered X-ray diffraction (X'Pert Philips) with CuK $\alpha$ radiation $(0.1540 \mathrm{~nm})$ polychomator beam in the $2 \theta$ scan range 20 $80{ }^{\circ} \mathrm{C}$ was carried to confirm the polymorphs of the composite materials from the SAED patterns. A step size and step time of $0.0170(2 \theta)$ and $87.63 \mathrm{~s}$ was used at $40 \mathrm{kV}$ and $40 \mathrm{~mA}$ instrument power settings. Raman data were recorded for further confirmation of the polymorphs of the composites. A Raman spectrometer, Raman Micro 200, PerkinElmer precisely used with a single monochromator, a holographic notch and a cooled TCD. The samples were exposed for $4 \mathrm{~s}$ during excitation using the $514.5 \mathrm{~nm} \mathrm{Ar}^{+}$line. The Raman instrument was equipped with video imaging which permitted the image capture of the nanocomposites.

The Brunauer-Emmett-Teller surface area $\left(S_{\mathrm{BET}}\right)$ of the NPs was determined by nitrogen adsorption in a Micrometric ASAP 2020. The samples were degassed at $100{ }^{\circ} \mathrm{C}$ for $12 \mathrm{~h}$ prior to analysis. Isotherm evaluation was performed at $\sim 77 \mathrm{~K}$, and pore volume analysis was determined at $\left(P / P_{\mathrm{o}}\right)$ of $\sim 0.98$.
Optical properties were recorded to understand on which part of the electromagnetic spectrum do the composites absorb and to elucidate the electronic structure of the composite materials using diffuse reflectance spectroscopy (DRS) and photoluminescence spectroscopy (PL). The UV-Vis spectra was recorded on a Shimadzu UV-2450 UV-Vis spectrophotometer, where $\mathrm{BaSO}_{4}$ was used as a reference. The photoluminescence spectra were recorded using a LS 45 fluorescence spectrometer (PerkinElmer, precisely) at $242 \mathrm{~nm}$ wavelength.

Electrochemical measurements were conducted using Metro Autolab: Nova 2.0 potentiostat in a standard three electrode system using $\mathrm{Ag} / \mathrm{AgCl}$ as a reference electrode, a Pt wire as a counter electrode and a working electrode fabricated using fluorine-doped tin oxide (FTO) glass with NPs pasted on the fluorine-doped side. The working electrodes were prepared by mixing the synthesized NPs with polyvinylidene fluoride (PVDF) in a ratio $10: 1$ respectively in $1 \mathrm{~mL} N$-methylpyridine (NMP). The PVDF was used as a binder and the NMP as a solvent to form a homogeneous slurry. The slurry was then drop casted on the FTO glass and allowed to dry overnight at room temperature. Electrical connection to the potentiostat was facilitated by attaching a copper wire using silver paste which was allowed to dry in air for $24 \mathrm{~h}$ at room temperature. The electrochemical impedance spectroscopy (EIS) spectra was conducted at a frequency range of $100 \mathrm{kHz}$ to $0.1 \mathrm{~Hz}$ at an AC voltage of $10 \mathrm{mV}$ rms vs. $E_{\text {ref }}$.

The quantitative surface sensitive elemental analysis, valence band measurements were done using X-ray photoelectron spectroscopy (XPS). Measurement of XPS was carried out with a Thermo spectroscope, model ESCAlab 250Xi using a monochromator $\mathrm{Al} \mathrm{K} \alpha(1486.7 \mathrm{eV})$ as an excitation source at a working pressure of $<10^{-8} \mathrm{mBar}$.
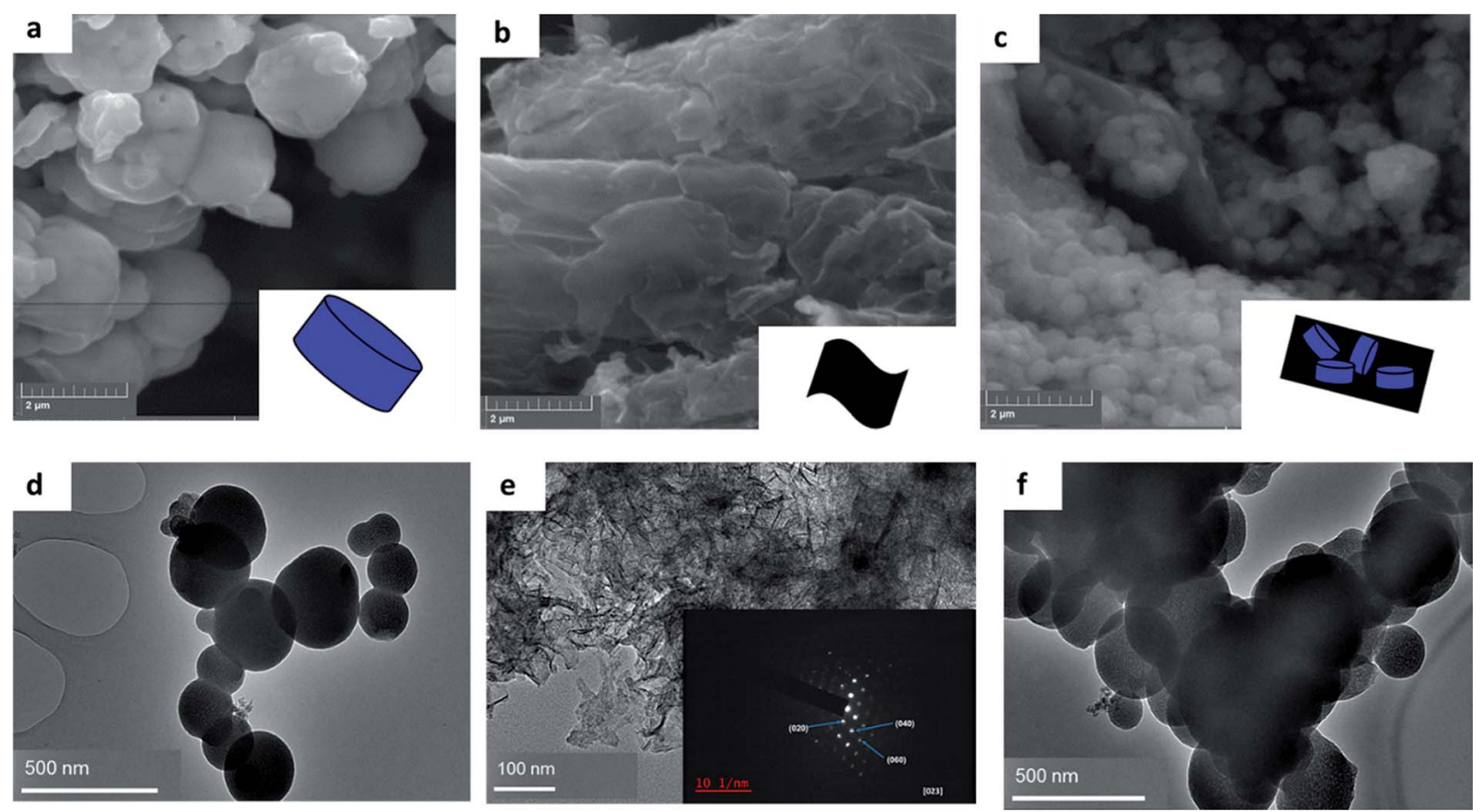

Fig. 1 SEM images of (a) MIL-125(Ti), (b) FLBP (c) 4\%BpMIL and TEM images of (d) MIL-125(Ti), (e) FLBP (f) 4\%BpMIL. 


\section{Results and discussion}

The texture and structural morphology of the as-synthesized pristine materials and optimum composite were observed via SEM and TEM imagery presented in Fig. 1. The images presented in Fig. 1(a) show the SEM image of MIL-125(Ti) which displayed circular oriented blocks. The sheet-like morphology of black phosphorus is shown in Fig. 1(b) and SEM imagery similar to the one reported has been presented before in literature, which confirmed the successful exfoliation of few layer black phosphorus. ${ }^{5}$ Fig. 1(c) presents the SEM images of the optimum composite ( $4 \% \mathrm{BPMIL}$ ) which clearly displayed the coexistence of circular blocked MIL-125(Ti) and sheet-like FLBP. The TEM pictorial illustrations are shown in Fig. 1(d-f) and they just re-affirm the individual morphologies of the pristine materials and the subsequent optimum nanocomposite. The SEM-EDS of the pristine and composite materials are presented Fig. S1-S5, $\uparrow$ which highlighted especially for the nanocomposites, the presence of the elements: titanium, oxygen, carbon and phosphorus. The morphologies of the 6\%BpMIL and $12 \%$ BpMIL composites are shown via SEM and TEM imagery presented in Fig. $\mathrm{S} 6$ and $\mathrm{S} 7 \uparrow$ these composites also exhibited circular blocks and sheet-like morphology comparable to $4 \%$ BpMIL confirming the presence of MIL-125(Ti) and $\mathrm{BP}$ respectively.

The polymorphs of the as-synthesized nanocomposites were elucidated through X-ray diffraction (XRD) presented in Fig. 2. The XRD patterns of MIL-125(Ti) were similar to one reported recently, ${ }^{23}$ demonstrating the successful synthesis of this $\mathrm{Ti}$ oriented MOF.

The diffraction peaks of FLBP depicted in (e) at $16^{\circ}$ and $34^{\circ}$ are indexed to (020), (040) respectively (JCPDS no. 73-1358), suggesting successful exfoliation of FLBP., ${ }^{5,24}$ This also confirmed the orthorhombic structure of FLBP as presented by the SAED patterns from TEM imagery (Fig. 1(e)). Compared to MIL-125(Ti) and exfoliated BP, the composites exhibited MIL125 and FLBP peaks which are denoted by the $(*)$ and (\#) respectively, indicating that a heterojunction was indeed successfully fabricated. The peaks $(*)$ of MIL-125(Ti) in the

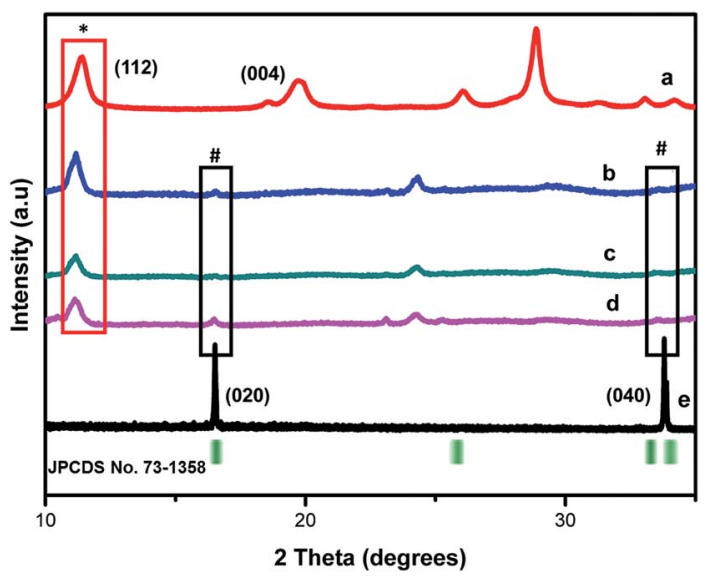

Fig. 2 XRD patterns of (a) MIL-125(Ti) (b) 4\%BpMIL (c) 6\%BpMIL (d) $12 \%$ BpMIL and (e) FLBP. composites became more intense as the loading of MIL125(Ti), suggesting the MIL-125(Ti) becomes dominant as the loading is increased. The appearance of new peaks between $22-26^{\circ}$ may infer a new polymorph as the heterostructure was formed.

To probe the bond vibration of the pristine materials and the subsequent composites, Raman spectroscopy was conducted (Fig. 3). The teraphthalic acid ligand makes MIL-125(Ti) Raman active and this evidenced by the bands indexed at 1618, 1455, 1181 and $1138 \mathrm{~cm}^{-1}$ which relate to the in-plane vibrational modes of the aromatic rings, whereas bands centered 865 and $641 \mathrm{~cm}^{-1}$ represent the vibrational modes of the $\mathrm{C}-\mathrm{H}$ and $\mathrm{C}=\mathrm{C}$ bonds of the aromatic rings. ${ }^{10,21,25}$ The variation for the Raman peaks further affirmed the successful synthesis of MIL-125(Ti). The Raman bands indexed at 363,444 and $462 \mathrm{~cm}^{-1}$ corresponding to $\mathrm{A}_{\mathrm{g}}^{1}, \mathrm{~B}_{2 \mathrm{~g}}$ and $\mathrm{A}_{\mathrm{g}}^{2}$ Raman modes of FLBP as shown in Fig. 3(B). ${ }^{26-28}$ This confirmed that bulk BP was successfully exfoliated and it had a two dimensional layered structure. New peaks for the composite, a fusion of FLBP and MIL-125(Ti) complimented the data from XRD suggesting the formation of the heterojunction. Fig. 3(C) reveals that there was a slight overlap of the MIL-125(Ti) band with the $B_{2 g}$ and $A_{g}^{2}$ bands of FLBP at around 444 to $462 \mathrm{~cm}^{-1}$ highlighting the co-existence of FLBP and MIL-125(Ti) in the hybrids. The Raman images displaying the morphology of the synthesized nanocomposites are presented in Fig. S8. $\dagger$

The $\mathrm{N}_{2}$ adsorption-desorption isotherms of the nanocomposites are presented in Fig. 4 and the full data is depicted in Table S1. $\dagger$ The synthesized photocatalysts exhibited type IV isotherm which is characteristic of mesoporous nanoparticles. The isotherms also displayed $\mathrm{H} 2$ hysteresis loops indicating that the nanocomposites exhibit highly regular pore structures which may be attributed to the framework of MIL-125(Ti). The BET surface area of pristine MIL-125(Ti) is $285 \mathrm{~m}^{2} \mathrm{~g}^{-1}$, whereas the $4 \%$ BpMIL exhibited the lowest surface area of the hybrid nanocomposites. The decrease of the surface area may be attributed to the blockage of the MIL-125(Ti) pores by FLBP. This is further confirmed by the pore size and pore volume data which shows a reduction for all the nanocomposites compared to pristine MIL-125(Ti).

To confirm the elemental composition, chemical state and the formation of the composite, XPS analysis was conducted. Fig. $5(\mathrm{a}-\mathrm{d})$ depicts the XPS spectra of $4 \%$ BpMIL together with the deconvoluted peaks of Ti, $\mathrm{C}$ and $\mathrm{O}$. The full survey spectra of FLBP is shown in Fig. S9. $\uparrow$ The full survey spectra of the optimum composite in Fig. 5(a) reveals the presence of Ti, C and $\mathrm{O}$, however the presence of $\mathrm{P}$ was not observed which may attributed to MIL-125(Ti) being dominant in the composite. The P element was observed in the full spectra of FLBP shown in Fig. S9, $\dagger$ however it also appeared as a weak peak, which might explain why it disappears when MIL-125(Ti) is hybridised with FLBP. Fig. 5(b) presents the Ti 2p spectra which displays peaks centered at 458.5 and $464.4 \mathrm{eV}$, corresponding to Ti $2 \mathrm{p}_{3 / 2}$ and Ti $2 \mathrm{p}_{1 / 2}$ indicating that titanium bounded to oxygen exists in +4 oxidation state for titanium-oxo-clusters. ${ }^{28}$ The C 1s spectrum in Fig. 5(c) was deconvoluted into three peaks attributed to $\mathrm{C}-\mathrm{O}$ at 284 and $286.0 \mathrm{eV}$, and $\mathrm{O}-\mathrm{C}=\mathrm{O}$ at 


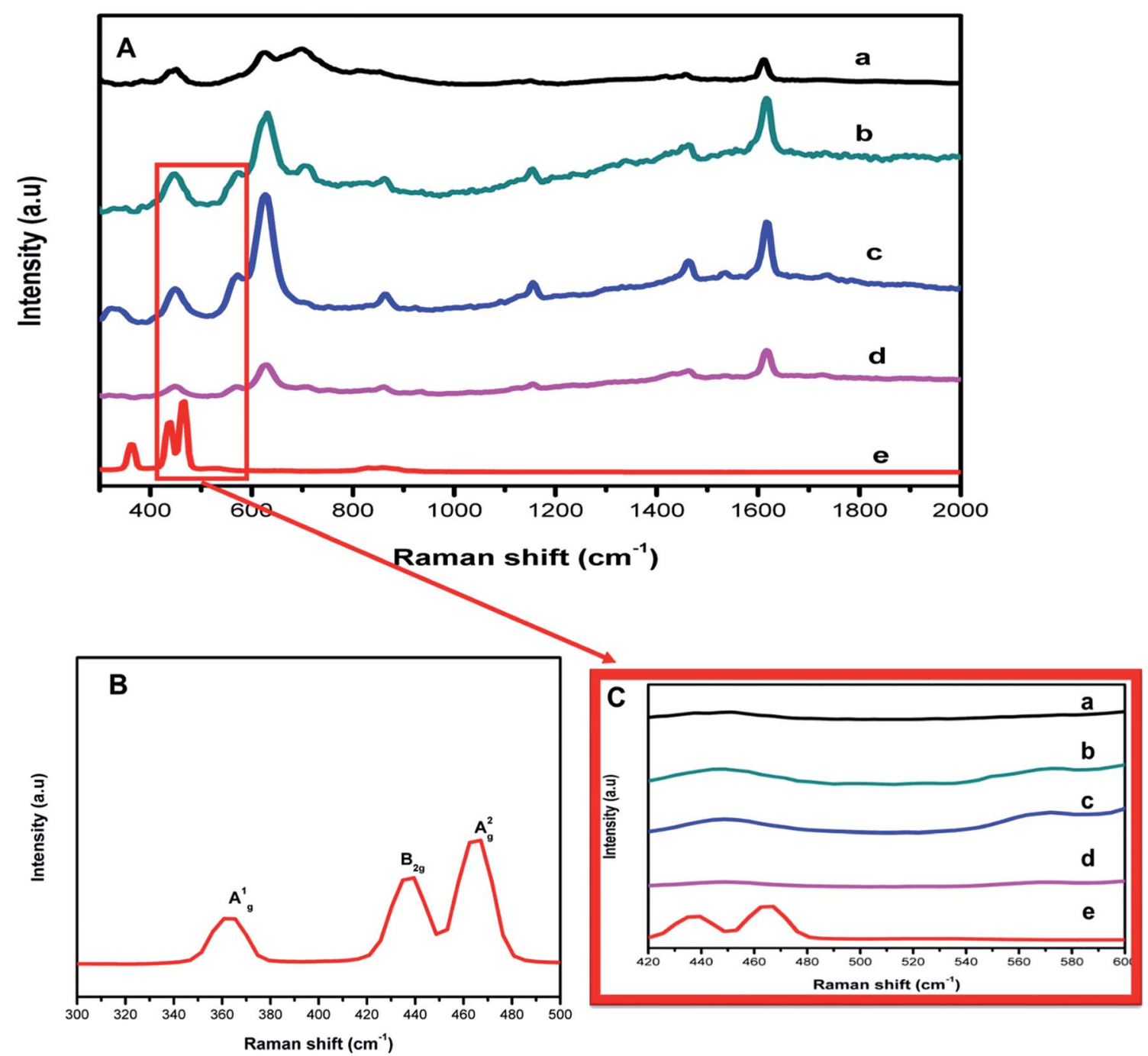

Fig. 3 (A) Raman bands of (a) MIL-125(Ti), (b) 4\%BpMIL, (c) 6\%BpMIL (d) 12\%BpMIL and (e) FLBP. (B) Zoomed image of the Raman spectra of FLBP (C) zoomed image showing Raman bands in the composites.

288.4 eV. These peaks confirmed that MIL-125(Ti) was formed instead of $\mathrm{TiO}_{2}$ Fig. $5(\mathrm{~d})$ depicts the $\mathrm{O} 1 \mathrm{~s}$ spectrum of $4 \%$ BpMIL. The binding energy values at 529.8, 531.5 and $533.3 \mathrm{eV}$ correspond to oxygen from $\mathrm{Ti}-\mathrm{O}$ bond in the titanium-oxocluster, oxygen in hydroxyl groups and oxygen in carbonyl groups respectively. ${ }^{19}$ These results showed a discrepancy because of the absence of phosphorus, however the VBXPS data presented in Fig. 6 validated the formation of the heterojunction between MIL-125(Ti) and FLBP, as a red-shift was observed for $4 \%$ BpMIL (2.24 eV) compared to pristine FLBP $(1.62 \mathrm{eV})$. These results revealed that the composite could be used for the evolution of oxygen from water. Furthermore this composite was expected to exhibit reduced recombination as XPS data gives more precise numerical values of valence band edge potentials of the composite.

UV-Vis DRS analyses (Fig. S10†) and Tauc plots (Fig. 7(a)) were used to probe the optical properties of the synthesized nanocomposites. These materials are indirect semiconductors, thus their band gaps were computed from DRS data by making use of (1).

$$
\alpha h \nu=A\left(h \nu-E_{\mathrm{g}}\right)^{n / 2}
$$

where $\alpha$ defines the absorption coefficient, $h$ the Plank's constant, $\nu$ the light frequency, $A$ is a constant, $E_{\mathrm{g}}$ represents the band gap and $n$ is determined based on the type of optical transition of a semiconductor. As stated above, NPs are indirect semiconductors, thus $n=2$.

The band gaps calculated from $h \nu(\mathrm{eV})=\frac{1240}{\lambda}$ on the Tauc plots are depicted in Fig. 7(a) with FLBP exhibiting the smallest band gap and $12 \%$ BpMIL the largest. It was observed that the band gap of the BPMIL intermediates reduced by $1.21 \mathrm{eV}$ or less after formation of the composites compared with pristine MIL125(Ti), hence inferring visible light response would be greatly enhanced upon application be it in water treatment or water splitting.

The composites exhibited an extended light absorption into the visible region and this was due FLBP serving as the visible light sensitizer. The combination of FLBP and MIL-125(Ti) to form heterojunctions leads to a red shift because of the 

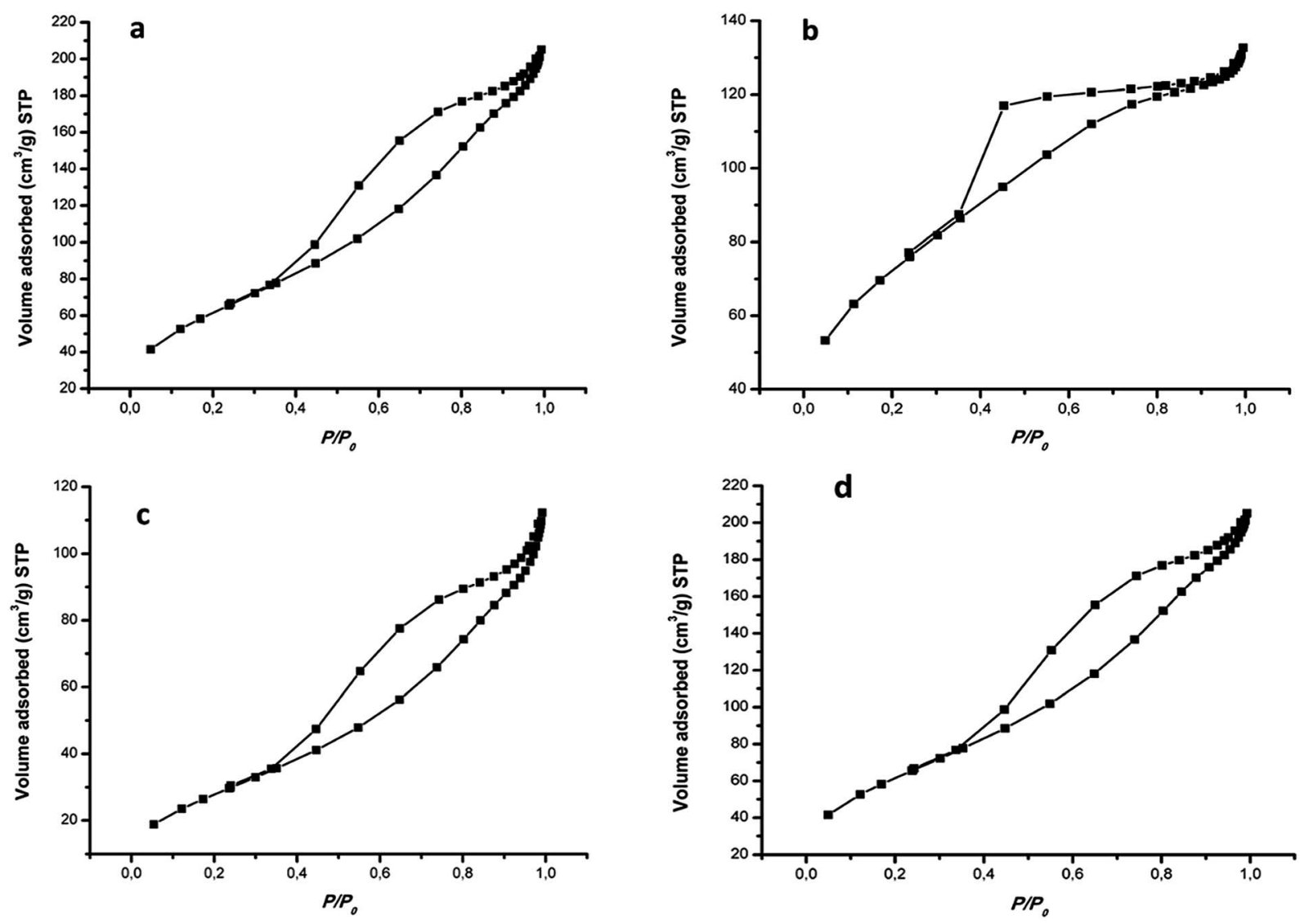

Fig. 4 Adsorption-desorption isotherms of (a) MIL-125(Ti), (b) 4\%BpMIL, (c) 6\%BpMIL and (d) 12\%BpMIL.

synergistic effect between both materials. The red shift in wavelength gives insight that these composites can be utilised for visible light photocatalysts. The UV-Vis spectra supported the synergistic role of FLBP and MIL-125(Ti) as it revealed that FLBP was visible light responsive, ${ }^{29}$ while MIL-125(Ti) is UV light responsive. ${ }^{30}$

To substantiate the claim that heterojunction formation minimizes recombination rate, PL studies were conducted and the spectra is depicted in Fig. 7(b). The peak intensities in the PL spectra give an idea about the recombination rate of a material. More precisely, a sharp peak signifies fast recombination and flat peak represents inhibited recombination probability. FLBP displayed a flat peak, MIL-125(Ti) had a very sharp peak. However after hybridising the two materials, it was evident that all the composites displayed a reduced peak compared to MIL-125(Ti), suggesting that the formation of heterojunctions significantly reduced the recombination rate in the composites. The $6 \%$ BpMIL composite displayed the lowest recombination of all the composites.

The valence and conduction band potentials were calculated using (2) and (3) respectively

$$
\begin{gathered}
E_{\mathrm{VB}}=X-E^{0}+0.5 E_{\mathrm{g}} \\
E_{\mathrm{CB}}=E_{\mathrm{VB}}-E_{\mathrm{g}}
\end{gathered}
$$

where $E_{\mathrm{VB}}$ is the valence band potential, $E_{\mathrm{CB}}$ is the conduction band potential, $E^{0}$ is the energy of free electrons $v s$. normal hydrogen electrode, which is approximately $4.5 \mathrm{eV}$ and $X$ is the semiconductor electronegativity, computed from the geometric mean of the absolute electronegativity of constituent atoms. ${ }^{31}$ The electronegativity of MIL-125(Ti) and FLBP were computed to be approximately $6.58 \mathrm{eV}$ and $4.67 \mathrm{eV}$ respectively. The band positions of the pristine materials (Fig. S11†) and the composites (Fig. S12 $\dagger$ ) supported the PL data as discussed before.

The staggered alignment of the CBs and VBs are tailored for the separation of photogenerated electron and holes upon visible light excitation. This should allow the transfer of electrons from the CB (FLBP) to the CB (MIL-125(Ti)), while holes are shuttled from the VB (MIL-125(Ti)) to VB (FLBP). Resulting in the electrons being separated from the holes, thus inhibiting the recombination rate, thus affirming the composites should exhibit enhanced visible photocatalytic activity because of reduced recombination probability. The enhanced visible light response of MIL-125(Ti) could be attributed to its photochromic performance which is closely related to the intervalence electron transfer bands caused by the optically triggered shuttle of electrons from $\mathrm{Ti}^{3+}$ to $\mathrm{Ti}^{4+}$ sites in the titanium oxo-clusters of MIL-125(Ti). ${ }^{14}$ While on the other end FLBP acts as visible light sensitizer, absorbing electrons and then migrating to the $\mathrm{Ti}^{4+}$ sites in the titanium oxo-clusters due to the staggered band 

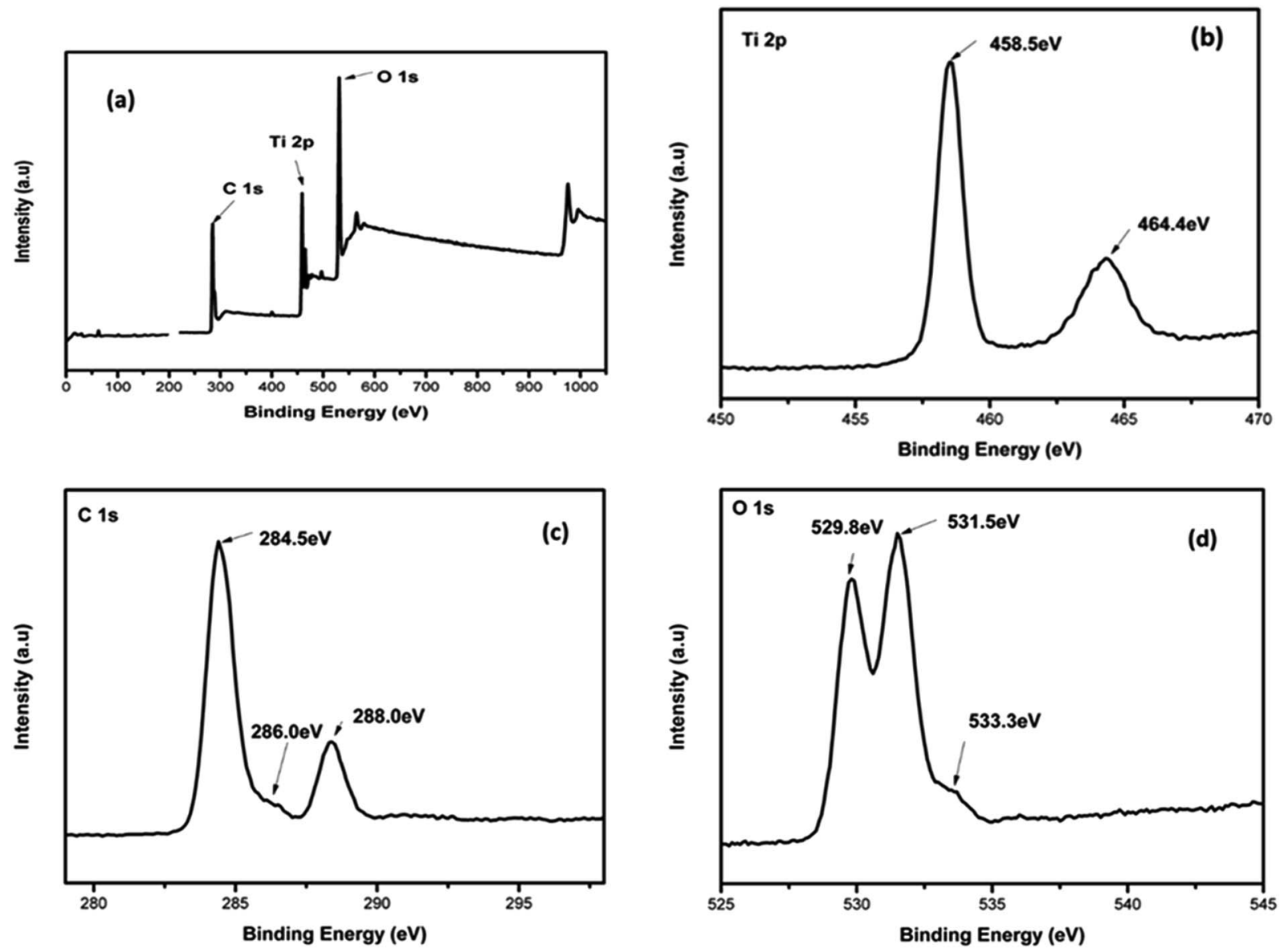

Fig. 5 The XPS spectra of 4\%BpMIL composite; (a) a full spectrum (b) Ti 2p spectrum (c) C 1s spectrum and (d) O 1s spectrum.

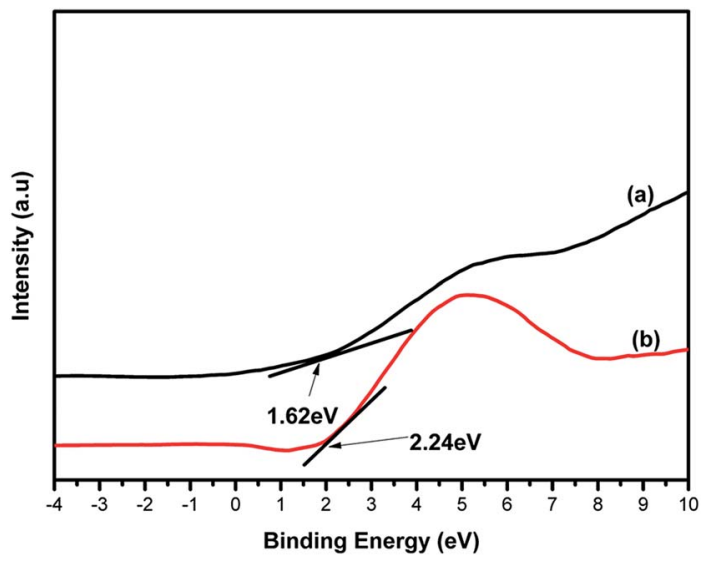

Fig. 6 The VBXPS spectra of (a) FLBP and (b) 4\%BpMIL.

alignment of the heterojunctions..$^{30}$ The $\mathrm{Ti}^{4+}$ is thus reduced to $\mathrm{Ti}^{3+}$ as a result of the intervalence electron transfer bands. In addition, the changes in energy levels of MIL-125(Ti) were possibly a result of the intervalence electron transfer band alignments in relation to the fermi levels of FLBP which are near the CB, when the Schottky contact was formed. A plausible mechanism for the intervalence electron transfer is given by (4) and (5)

$$
\begin{gathered}
\text { FLBP }+h \nu \rightarrow \operatorname{FLBP}\left(\mathrm{e}^{-}+\mathrm{h}^{+}\right) \\
\text {MIL-125(Ti) }+\mathrm{e}^{-} \rightarrow \mathrm{Ti}^{3+}-\text { MIL-125(Ti) }
\end{gathered}
$$

To validate the findings from photoluminescence studies, Mott-Schottky as deduced from the potentiostat were plotted using (6) to further validate the formation of the heterojunction.

$$
\frac{1}{C^{2}}=\frac{2}{\varepsilon \varepsilon_{0} e_{0} N_{\mathrm{D}}}\left(E-E_{\mathrm{FB}}-\frac{k T}{e_{0}}\right)
$$

where $\varepsilon$ is the permittivity of the semiconductor electrode, $e_{0}$ is the elementary charge, $N_{\mathrm{D}}$ is the donor density, $E$ the applied potential, $E_{\mathrm{FB}}$ the flat-band potential, $k$ is the Boltzmann constant, $T$ the temperature of operation and $C$ is the space charge capacitance.

The Mott-Schottky plots are depicted in Fig. 8(A) and complemented the results from the PL (Fig. 7(b)) fairly well. The gradient of the plot of $1 / C^{2}$ against $E$ gives insight into the recombination of charge carriers. ${ }^{31}$ A small gradient after heterojunction formation represents reduced probability of recombination of charge transporters. The data presented in Fig. 8 suggests that the recombination rate decreases in the following order MIL-125(Ti) $>12 \%$ BpMIL $>6 \%$ BpMIL $>4 \%$ BPMIL > FLBP as supported by the PL findings. Pristine MIL125(Ti) displayed a positive gradient which is characteristic of 

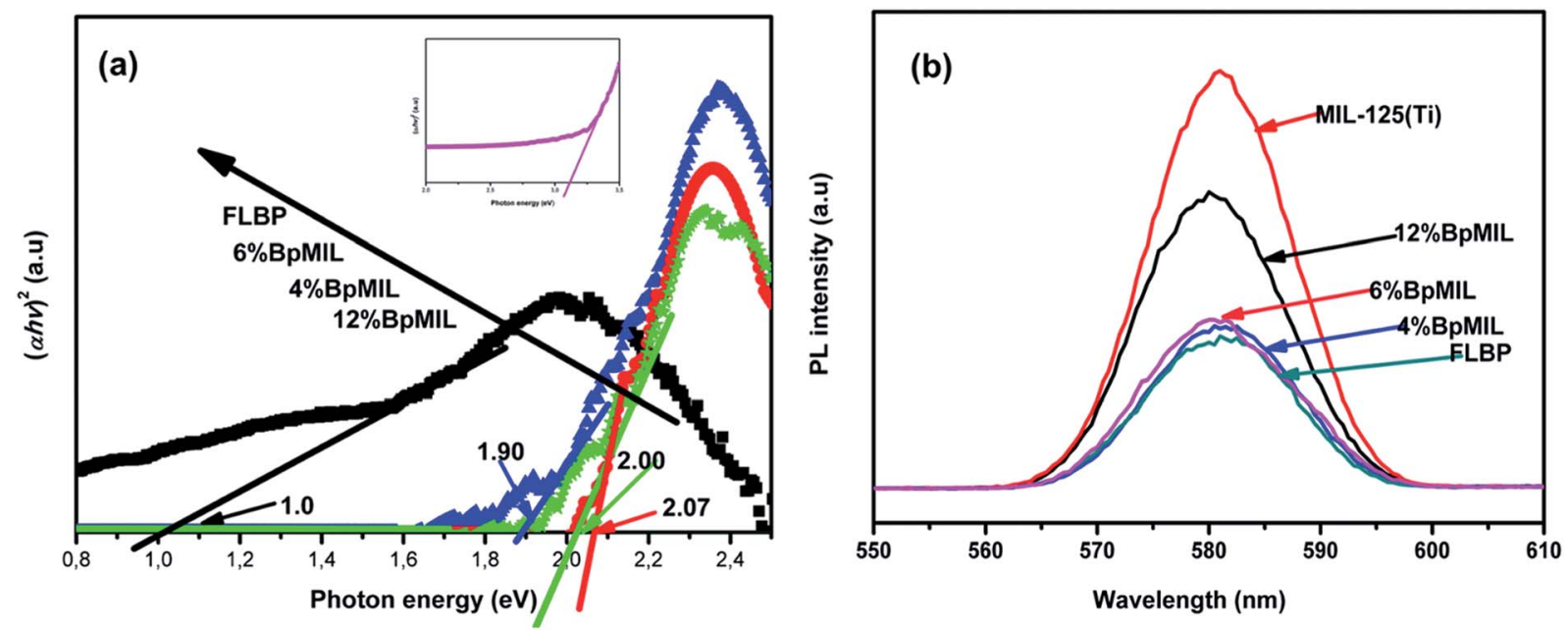

Fig. 7 (a) Tauc plots with MIL-125(Ti) (inset) and (b) PL spectra of synthesized NPs.

n-type semiconductors, however all the composites showed a negative slope which represents p-type semiconductivity.

Additionally, presented in Fig. 8(a-f), the flat band potentials $\left(V_{\mathrm{FB}}\right)$ of the pristine FLBP and MIL-125 were $-0.90 \mathrm{~V}$ and $0.85 \mathrm{~V}$ respectively, signifying that the CB of FLBP would be positioned above the CB of MIL-125(Ti) in a p-n heterojunction whereas the $4 \%$ BpMIL, 6\%BpMIL and 12\%BpMIL composites exhibited $-1.40 \mathrm{~V},-0.60 \mathrm{~V}$, and $-1.55 \mathrm{~V}$ respectively. The flat band potentials of the composite materials are more negative than that of FLBP, revealing that indeed the heterojunction
A
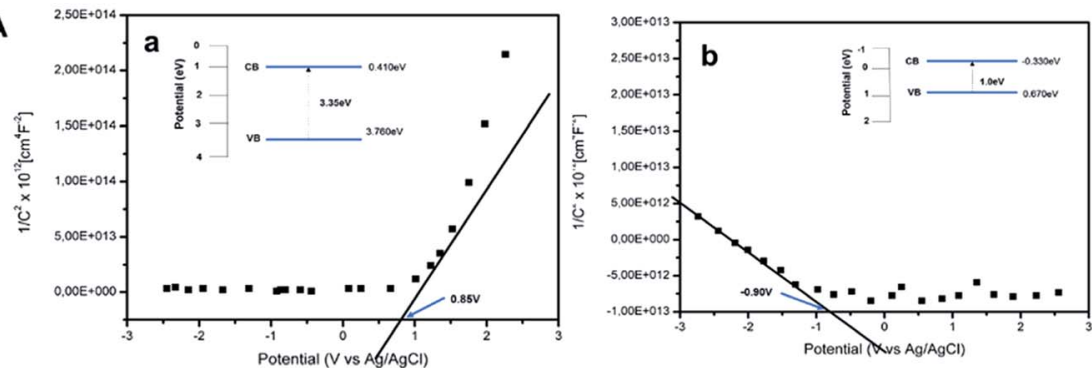
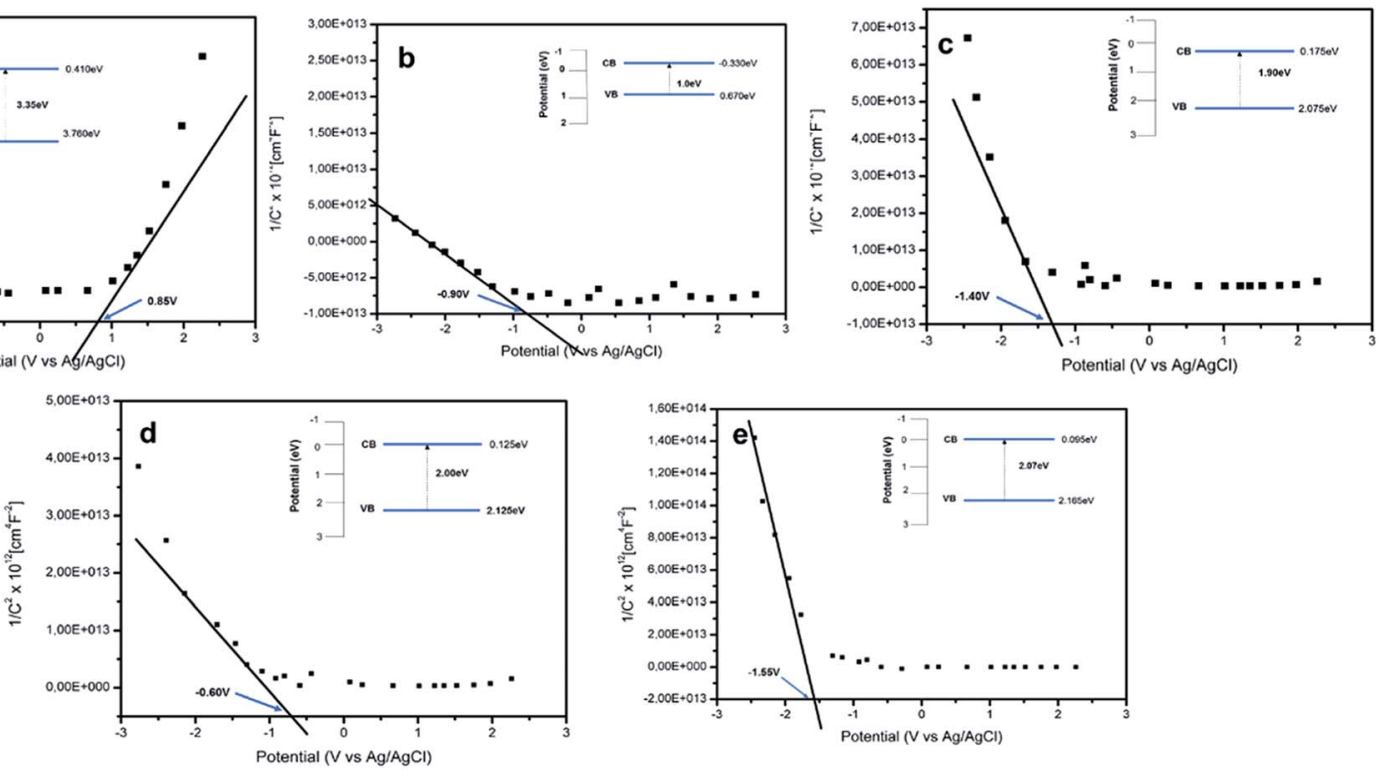

B

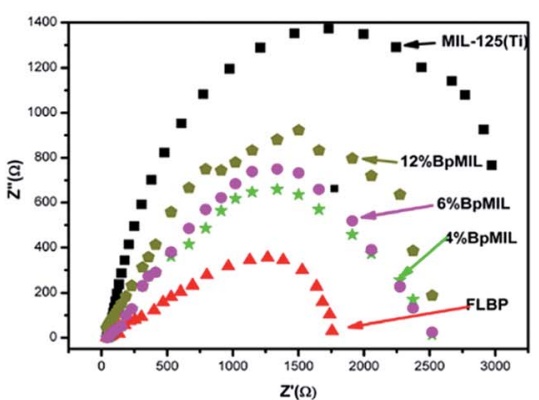

Fig. 8 (A) Mott-Schottky plots of (a) MIL-125(Ti), (b) FLBP (c) 4\%BpMIL, (d) 6\%BpMIL and (e) 12\%BpMIL and (B) Nyquist plots of MIL and composites. 
formation suppresses the recombination rate. ${ }^{32}$ Even though $6 \%$ BpMIL is more positive than FLBP, its negative flat-band potential still confirms that heterojunction formation inhibits recombination probability. The accompanying band positions are presented revealing the formation of the $\mathrm{p}-\mathrm{n}$ junctions between p-type FLBP and n-type MIL-125(Ti). It is worth noting that the composites conformed to the FLBP semiconductor behaviour because FLBP is an excellent charge transporter, which has an impact on the reduction of recombination rate and is thus, propitious for photocatalytic activity.

Electrochemical impedance spectroscopy (EIS) studied for the viewpoint of Nyquist plots was used to probe the interfacial interaction between the photoelectrode and the electrolytes. Nyquist plots give insight into the recombination rates by studying the diameter of the semi-circles formed between the imaginary $\left(Z^{\prime \prime}(\Omega)\right)$ and real $\left(Z^{\prime}(\Omega)\right)$ parts of complex impedance. The interpretation of semi-circle provides information about the recombination rate in the sense that a small semi-circle diameter signifies small charge transfer resistance of the working electrode and therefore low recombination probability. ${ }^{33}$

In other cases the Nyquist plot may have a semi-circle adjoined to another semi-circle or a nonvertical line at subsequent frequencies. The second semi-circle provides information on the roughness of the electrode. The first semi-circle represents the electrolyte resistance or the charge shuttle resistance whereas the non-vertical line signifies the ion transfer limitation in the electrolyte for porous electrode structures. ${ }^{33}$

The Nyquist plots of the pristine and composite materials are displayed in Fig. 8(B). The recombination rate was observed to decrease in the order MIL-125(Ti) > 12\%BpMIL > 6\%BpMIL > $4 \%$ BpMIL > FLBP, which is the same data sourced from the Mott-Schottky plots. 4\%BpMIL which was the optimum hybrid displayed the lowest charge resistance of all the composites which translates to the lowest recombination rate.

Furthermore, equivalence circuits and fitted circuits are presented in Fig. S13-S15† for the pristine materials and the optimum composite. The values of the charge transfer resistance $R$ were found to be $61.3 \Omega, 45.1 \Omega$ and $49.7 \Omega$ for MIL125(Ti), FLBP and 4\%BpMIL. This validates that heterojunction formation leads to efficient separation of charge carriers, however FLBP exhibited a lower $R$ value than the composite underlining the fact that FLBP is an excellent charge transporter. The findings from the Nyquist plots further affirmed the results from PL and Mott-Schottky plots. The optical and electrochemical studies confirmed the successful fabrication of a $\mathrm{p}-\mathrm{n}$ heterojunctions between FLBP and MIL125(Ti). From the PL data, Mott-Schottky plots and Nyquist plots, we deduced that the $4 \%$ BpMIL composite as a visible light responsive heterojunction material exhibited reduced recombination rate and the effectual separation of charge carriers.

\section{Conclusion}

A series of MIL-125(Ti)/FLBP composites were synthesized using a two-step hydrothermal and sonication method by varying the loading of MIL-125(Ti) while keeping the amount of FLBP constant. The results from different characterization data revealed that heterojunctions were successfully fabricated and they were all $\mathrm{p}-\mathrm{n}$ type which is beneficial for the separation of photo-induced charge carriers. Furthermore, the optical and electrochemical data showed that the $4 \%$ BpMIL composite had the lowest recombination rate and fastest interfacial transfer as a result of FLBP and the formation of the p-n junction. Evidence of the formation of the heterojunction was substantiated by XPS data which showed a red-shift for the VB in the optimum nanocomposite. The positive shift indicates that the photocatalyst can be applied in the evolution of oxygen from water as a more positive VB is suited for water splitting into oxygen. The fabrication of $2 \mathrm{D} / \mathrm{MOF}$ composites with new properties and applications can be utilized as visible light responsive materials.

\section{Conflicts of interest}

On behalf of all authors, the corresponding author states that there is no conflict of interest.

\section{Acknowledgements}

The authors would like to pass their sincere thanks to the following funders of the work; the authors would like to acknowledge the University of Johannesburg (URC) and Faculty of Science (FRC), National Research Foundation (Thuthuka), Centre for Nanomaterials Science Research (CNSR) and Eskom (TESP) for financial support.

\section{References}

1 A. Ismail and D. Bahnemann, Solar Energy Materials \& Solar Cells Photochemical splitting of water for hydrogen production by photocatalysis: A review, Sol. Energy Mater. Sol. Cells, 2014, 128, 85-101.

2 R. Marschall, Semiconductor composites: Strategies for enhancing charge carrier separation to improve photocatalytic activity, Adv. Funct. Mater., 2014, 24(17), 2421-2440.

3 I. Bannat, K. Wessels, T. Oekerumann, J. Rathousky, D. Bahanemann and $M$. Wark, Improving the Photocatalytic Performance of Mesoporous Titania Films by Modification with Gold Nanostructures, Chem. Mater., 2009, 21(8), 1645-1653.

4 A. Ibhadon and P. Fitzpatrick, Heterogeneous Photocatalysis: Recent Advances and Applications, Catalysts, 2013, 3(1), 189-218.

5 L. Wang, Q. Xu, J. Xu and J. Weng, Synthesis of hybrid nanocomposites of ZIF-8 with two-dimensional black phosphorus for photocatalysis, RSC Adv., 2016, 6(73), 69033-69039.

6 H. Dong, G. Zeng, L. Tang and C. Fan, An overview on limitations of $\mathrm{TiO}_{2}$-based particles for photocatalytic degradation of organic pollutants and the corresponding countermeasures, Water Res., 2015, 79, 128-146. 
7 H. Wang, L. Zhang, Z. Chen, J. Hu and S. Li, Semiconductor heterojunction photocatalysts: design, construction, and photocatalytic, Chem. Soc. Rev., 2014, 43(15), 5234-5244.

8 C. Zhang, L. Qiu, F. Ke, Y. Zhu, Y. Yaun, G. Xu and X. Jiang, A novel magnetic recyclable photocatalyst based on a coreshell metal-organic framework $\mathrm{Fe}_{3} \mathrm{O}_{4} @ M I L-100(\mathrm{Fe})$ for the decolorization of methylene blue dye, J. Mater. Chem. A, 2013, 1, 14329-14334.

9 M. Jin, X. Qian, J. Gao, J. Chen, D. Hensley, H. Ho, R. Percoco, C. Ritzi and Y. Yue, Solvent-Free Synthesis of CuO/HKUST-1 Composite and Its Photocatalytic Application, Inorg. Chem., 2019, 58(13), 8332-8338.

10 X. Yuan, H. Wang, Y. Wu and G. Zeng, One-pot self-assembly and photoreduction synthesis of silver nanoparticledecorated reduced graphene oxide/MIL-125 (Ti) photocatalyst with improved visible light photocatalytic activity, Appl. Organomet. Chem., 2016, 30(5), 289-296.

11 Y. Lee, J. Kim and W. Ahn, Synthesis of metal-organic frameworks: A mini review, Korean J. Chem. Eng., 2013, 30(9), 1667-1680.

12 S. Dhaka, R. Kumar, A. Deep, M. Kurude, S. Woo Ji and B. Jeon, Metal-organic frameworks (MOFs) for the removal of emerging contaminants from aquatic environments, Coord. Chem. Rev., 2019, 380, 330-352.

13 K. Kumar, S. Maddila, S. Babu and S. Jonnalagadda, Inorganica Chimica Acta A review on contemporary Metal-Organic Framework materials, Inorg. Chim. Acta, 2016, 446, 61-74.

14 M. Dan-hardi and C. Serre, A New Photoactive Crystalline Highly Porous Titanium (IV) Dicarboxylate, J. Am. Chem. Soc., 2009, 131(31), 10857-10859.

$15 \mathrm{~K}$. Wang and J. Chen, Extended structures and physicochemical properties of uranyl-organic compounds, Acc. Chem. Res., 2011, 44(7), 531-540.

16 M. Rahman, P. Kwong, K. Davey and S. Qiao, 2D Phosphorene as a Water Splitting Photocatalyst: A Review of Fundamentals to Applications, Energy Environ. Sci., 2016, 9(3), 709-728.

17 W. Lei, G. Liu, J. Zhang and M. Liu, Black phosphorus nanostructures: recent advances in hybridization, doping and functionalization, Chem. Soc. Rev., 2017, 46(12), 3492-3509.

18 J. Brent, N. Savjani, E. Lewis, S. Haigh, D. Lewis and P. O'Brien, Production of few-layer phosphorene by liquid exfoliation of black phosphorus, Chem. Commun., 2014, 50, 13338-13341.

19 S. Lin, Y. Chui, Y. Li and P. Lau, Liquid-phase exfoliation of black phosphorus and its applications, FlatChem, 2017, 2, $15-37$.

20 A. Khandelwal, K. Mani, M. Karigerasi and I. Lahiri, Phosphorene - The two-dimensional black phosphorous: Properties, synthesis and applications, Mater. Sci. Eng., B, 2017, 221, 17-34.
21 Z. Yang, J. Ding, J. Feng, C. He, Y. Li, X. Tong, X. Niu and H. Zhang, Preparation of $\mathrm{BiVO}_{4} / \mathrm{MIL}-125(\mathrm{Ti})$ composite with enhanced visible-light photocatalytic activity for dye degradation, Appl. Organomet. Chem., 2018, 32(5), 1-10.

22 M. Zhu, Z. Sun, M. Fujitsuka and T. Majima, Z-Scheme Photocatalytic Water Splitting on a 2D Heterostructure of Black Phosphorus/Bismuth Vanadate Using Visible Light, Angew. Chem., Int. Ed., 2018, 57(8), 2160-2164.

23 P. Hlophe, L. Mahlalela and L. Dlamini, A composite of platelet-like orientated $\mathrm{BiVO}_{4}$ fused with MIL-125 (Ti): Synthesis and characterization, Sci. Rep., 2019, 9, 1-12.

24 P. Qiu, C. Xu, N. Zhou, H. Chen and F. Jiang, Metal-free black phosphorus nanosheets-decorated graphitic carbon nitride nanosheets with $\mathrm{C}-\mathrm{P}$ bonds for excellent photocatalytic nitrogen fixation, Appl. Catal., B, 2018, 221, 27-35.

25 P. Banerjee, D. Lobo, R. Middag, W. Ng, M. Shaibani and M. Majumder, Electrochemical Capacitance of Ni-Doped Metal Organic Framework and Reduced Graphene Oxide Composites, ACS Appl. Mater. Interfaces, 2015, 7(6), 3655-3664.

26 D. Hanlon, C. Backer, E. Doherty, C. Cucinotta, N. Berner, C. Boland, K. Lee, A. Harvey, P. Lynch, Z. Gholavmvand, S. Zhang, K. Wang, G. Moynihan and J. Coleman, Liquid exfoliation of solvent-stabilized few-layer black phosphorus for applications beyond electronics, Nat. Commun., 2015, 6, 8563-8583.

27 V. Eswaraiah, Q. Zeng, Y. Long and Z. Liu, Black Phosphorus Nanosheets: Synthesis, Characterization and Applications, Small, 2016, 12(26), 3480-3502.

28 L. Wang, Q. Xu, J. Xu and J. Weng, Synthesis of hybrid nanocomposites of ZIF-8 with two-dimensional black phosphorus for photocatalysis, $R S C A d v$., 2016, 6(73), 69033-69039.

29 M. Akhtar, G. Anderson, R. Zhao, A. Alruqi, J. Mroczkowska, G. Summanasekera and J. Jasinski, Recent advances in synthesis, properties, and applications of phosphorene, npj 2D Mater. Appl., 2017, 1, 5.

30 H. Wang, X. Yaun, Y. Wu, G. Zeng, X. Chen, J. Leng, Z. Wu, L. Jiang and H. Li, Facile synthesis of amino-functionalized titanium metal-organic frameworks and their superior visible-light photocatalytic activity for $\mathrm{Cr}(\mathrm{VI})$ reduction, $J$. Hazard. Mater., 2015, 286, 187-194.

31 K. Ye, X. Yu, Z. Qiu, X. Lu and Y. Zhang, Facile synthesis of bismuth oxide/bismuth vanadate heterostructures for efficient photoelectrochemical cells, RSC Adv., 2015, 5(43), 34152-34156.

32 M. Radecka, M. Rekas, A. Trenczek-zajac and K. Zakrzewska, Importance of the band gap energy and flat band potential for application of modified $\mathrm{TiO}_{2}$ photoanodes in water photolysis, J. Power Sources, 2008, 181(1), 46-55.

33 B. Mei, O. Munteshari, J. Lau, B. Dunn and L. Pilon, Physical Interpretations of Nyquist Plots for EDLC Electrodes and Devices, J. Phys. Chem. C, 2018, 122(1), 194-206. 